\title{
Activités
}

7-2 | octobre 2010

Varia

\section{Francis Ginsbourger (2010). Ce qui tue le travail}

Paris : Michalon, $187 \mathrm{p}$.

\section{Pascal Ughetto}

\section{(2) OpenEdition}

Journals

Édition électronique

URL : http://journals.openedition.org/activites/2396

DOI : $10.4000 /$ activites. 2396

ISSN : 1765-2723

Éditeur

ARPACT - Association Recherches et Pratiques sur les ACTivités

Référence électronique

Pascal Ughetto, «Francis Ginsbourger (2010). Ce qui tue le travail », Activités [En ligne], 7-2 I octobre 2010, mis en ligne le 15 octobre 2010, consulté le 23 septembre 2020. URL : http://

journals.openedition.org/activites/2396; DOI : https://doi.org/10.4000/activites.2396

\section{(c) (i) (9)}

Activités est mis à disposition selon les termes de la licence Creative Commons Attribution - Pas d'Utilisation Commerciale - Pas de Modification 4.0 International. 


\section{Analyse d'ouvrage}

\section{par Pascal Ughetto}

Université Paris-Est, LATTS

pascal.ughetto@univ-paris-est.fr

\section{Francis Ginsbourger (2010). Ce qui tue le travail, Paris: Michalon, 187 p.}

Le travail a connu, avec les vagues de suicides professionnels, un retour fracassant dans l'actualité. Il fallait une telle intensité dramatique, un tel effet théâtral, pour ébranler les certitudes ou, au moins, obtenir l'attention des décideurs d'entreprises et politiques sur les mauvais traitements qui ont été imposés depuis plusieurs années au travail et aux salariés. Avec quelle capacité des décideurs à entendre le message? L'ouvrage de Francis Ginsbourger s'intéresse à cette surdité qui s'est installée au fil des décennies passées à l'encontre du travail, ainsi qu'aux sources de cette surdité au sein de la gestion. Sa thèse consiste à faire comprendre que, dans les catégorisations dont usent les politiques publiques et d'entreprises pour mettre en forme les problèmes de gestion à surmonter, le travail sort vaincu au détriment d'autres catégories, comme l'emploi. L'autre volet de la thèse est que la crise des suicides a vu se répandre des catégories, autour de la souffrance au travail et des risques psychosociaux, qui n'aident pas davantage à ouvrir les réflexions sur ce qu'est le travail: un espace d'apprentissage autour des produits et des procédés où se jouent le développement personnel des salariés et la création de valeur.

Là où beaucoup se lamentent sur les effets de la financiarisation, F. Ginsbourger demande de prendre le temps de comprendre ce qu'est la gestion des entreprises. Amplement inspirée par les théories du Centre de gestion scientifique de l'Ecole des Mines et du Centre de recherche en gestion de l'Ecole Polytechnique, son approche est que la gestion fonctionne à travers des catégories (d'appréhension du réel) servant à réduire du complexe à du " gérable » et que ces catégories, synthétisant ainsi le réel, produisent des effets qui vont au-delà de ce qui est envisagé, s'autonomisent, vivent leur vie et, éventuellement, se retournent contre les intentions initiales. Les catégories aident à voir des problèmes, sous un certain angle; elles empêchent aussi du même coup de voir les autres angles et tout bonnement de concevoir des problèmes. L'avantage se mue en revers: en offrant un prêt-à-penser, elles peuvent aussi empêcher «l'intelligence des situations vives auxquelles sont confrontés les acteurs » (p. 12).

Dans la gouvernance d'entreprise ou les modes d'action de l'Etat devenus dominants, le caractère nécessairement réducteur des catégories de gestion se montre d'autant plus source d'effets pervers que, à coups de pilotage par indicateurs et de communication politique, «l'action se veut centralisée, contrôlable à distance et propice aux effets d'affichage. » (p. 13). Le travail en est directement victime: pour piloter à distance, les grandes organisations en viennent, selon les termes de l'auteur, à « sur-gérer », synonyme d'une méfiance vis-à-vis des salariés: «elles instrumentent d'autant plus les processus qu'elles reconnaissent et valorisent peu la compétence » (p. 15). La compétence est entendue ici dans la lignée de ce qu'enseignent à son sujet Yves Lichtenberger et Philippe Zarifian, comme une aptitude manifestée dans les situations problématiques de travail - situations non totalement réglées par une programmation préalable -, à rendre des arbitrages, à prendre des initiatives et la responsabilité à l'égard de ces situations.

Les catégories de gestion se forment pour aider à surmonter des épreuves qui ne trouvent pas une solution spontanée et sur laquelle des controverses font entrevoir plusieurs options gestionnaires possibles, plusieurs manières de rendre le problème gérable. Ces dernières années ont vu se réduire l'espace accordé à la controverse et les acteurs s'installer dans la représentation appauvrie postulée par les catégorisations ayant cours. Ainsi, alors que les activités de service et la relation de service auraient dû interroger les catégories de gestion, les réflexes des catégories héritées de l'industrie 
l'ont largement emporté, avec là encore des répercussions directes pour le travail, confronté « au rouleau compresseur de la rationalisation industrialiste, comme si les identités professionnelles et l'expérience étaient des excédents de bagage » (p. 18). Cette formule imagée signifie que la performance productive et économique est considérée comme n'empruntant que très peu à la contribution qu'apportent les salariés, leur expérience intime des situations productives et les réponses qui sont les leurs en dehors des systèmes normés.

Là se trouve l'explication des drames personnels exprimés par les salariés jusque dans les suicides: plus qu'on ne peut le soupçonner, le fait de « perdre son métier» (p. 18) est un processus destructeur. Les premiers responsables sont alors « les gestions à distance » qui l'ont emporté coûte que coûte « sur les signaux qui provenaient du terrain » (ibid.).

C'est là que, pour l'auteur, les réponses gestionnaires aux suicides, la création de dispositifs « pour, par exemple, « dépister » les personnes supposées « fragiles » (pp. 18-19), ajoutent à l'existant sans inciter à une controverse sur les catégories de gestion pertinentes. L'étiquette des « risques psychosociaux » et la psychologisation de la souffrance au travail ne conduisent pas les décideurs et gestionnaires à interroger les leviers recherchés pour la création de valeur et à percevoir la contribution professionnelle. Le travail et sa contribution à l'efficacité, c'est là un prisme qui s'est effacé des politiques publiques et de la gestion d'entreprise. Ce prisme est celui qui prête aux salariés une volonté de compétence, un souci d'agir et de bien faire, et qui permet de porter l'attention sur ce qu'ils endurent quand ils sont exposés à des situations compliquées, par exemple de violence, sans le soutien de ressources comme l'expérience individuelle et collective.

Le premier chapitre montre comment s'est imposée la catégorie « emploi » au détriment de celle du travail à partir des années 1990: créer des emplois, défendre les emplois, comme si cela était indépendant des apprentissages développés dans le travail. Dans un tel raisonnement, les organisations, les fonctionnements organisationnels, les groupes professionnels et leur rôle dans la production d'identités et d'efficacité n'importent plus. Depuis cette époque, politiques d'emploi et gestion ont en commun des catégories, comme les bas niveaux de qualification, dont la problématique de gestion est la « remise à niveau », à la limite sans lien recherché avec l'acquisition, la reconnaissance, la transmission des savoir-faire d'expérience. L'autre est la réduction du coût du travail.

Le chapitre 2 revient sur l'abandon, par les politiques des années 1990 et 2000, du lien entre l'emploi et la qualité de l'organisation. Dans les entreprises, les DRH gèrent des effectifs, de la masse salariale, tout sauf du travail. Mais que reste-t-il de l'organisation? «On est arrivé à un tel point de dilution de l'action organisatrice que le niveau propre de l'organisation du travail, en tant que niveau distinct de l'organisation de la production (choix technologiques, de faire ou de faire faire) et de l'organisation générale de l'entreprise (celle qui traduit la stratégie, se reflète dans l'organigramme et la caractérisation du « cœur de métier ») a tendance à ne plus faire l'objet d'une gestion à part. Il n'y a plus de bureaux des méthodes, de spécialistes dédiés à une fonction globale et unifiée d'organisation. » (p. 58).

Le chapitre 3 fait comprendre d'où les thèses défendues par l'auteur lui sont venues. L'auteur y relate son expérience, entre 1998 et 2002, d'expert mandaté par les institutions représentatives du personnel à l'occasion de restructurations. L'intervenant qu'il est se sent pris dans un jeu entre la direction et les organisations syndicales où toutes manifestent une préférence pour des « guérillas verbales » autour de l'emploi. « Nécessités imposées par le marché », d'un côté, versus stigmatisation des « licenciements boursiers » de l'autre. Dans les deux cas, les raisonnements gestionnaires et le travail passent à la trappe. À s'envoyer ainsi des noms d'oiseaux, on passe à côté de tout un ensemble bien plus concret: « des analyses fouillées des arrêts répétés de la chaîne; des effets différés d'externalisation d'une maintenance que personne ne maîtrisait plus depuis la mise en préretraite du précédent chef d'atelier; le constat que l'on risquait, en supprimant les antennes commerciales régionales, d'affaiblir un maillon du réseau, pourtant proche des prescripteurs sur des marchés affichés comme stratégiques...» (p. 70). Il faut dire que, même du côté des directions, outre que l'organisation du travail est considérée comme une prérogative de l'employeur qui ne se discute pas, il serait difficile 
de le faire: il n'y a plus d'organisateur en titre, les centres de décision sont fuyants.

Pour se sortir de ces dialogues de sourds autour de l'emploi et de sa défense, l'auteur a choisi de ne plus accepter d'intervenir que sur la base d'un mandatement commun par les directions et les organisations de salariés. « Avec l'idée de relier, de « recoudre » et de faire se confronter l'analyse de l'activité de travail faite avec les uns et l'analyse économique et gestionnaire faite avec les autres. » (p. 73). Ce qui, selon lui, change tout: l'emploi n'occasionnait que « le choc des monologues » (p. 74), avec d'inévitables montées en généralité (les licenciements sont le mal, la défense de l'emploi est le bien); autour de l'analyse partagée du travail, est amené à vivre « le visage plus familier des protagonistes qui, bon an mal an, coopèrent au quotidien dans le travail » (p. 74).

On parle alors de compétence, mais non à la façon de l'ingénierie RH, que l'auteur voit comme un consensus mou qui s'est écarté des fonctionnements productifs. La compétence dont parle l'auteur est une notion ambitieuse: elle a partie liée avec la réalité des situations de production où, quotidiennement, le travail voit, pour s'accomplir, des initiatives se prendre, par-delà la consigne. Les restructurations, nous dit, cependant, l'auteur, ne sont pas un bon moment pour faire parler de la compétence: les salariés, même les cadres, ont volontiers tendance à se réfugier derrière une description de leur travail où ils sont censés ne faire qu'exécuter et une crainte de ne pas savoir faire autre chose.

Le chapitre 4 revient alors sur les voies empruntées depuis les années 1990 par les catégorisations: des politiques publiques à la gestion des entreprises, se sont imposées des catégories ( «bas niveaux de qualification », « coût du travail », « lutte contre l'exclusion », « partage du travail ») qui, prétendant dénoncer des injustices, ont créé des outils incitant ceux présentés comme fragiles, dominés, souffrants, à se camper en victimes. Au lieu de leur donner des instruments pour opposer d'autres catégorisations leur permettant d' « être acteurs de leur destinée, de se sentir partie prenante du problème posé, de faire la part des choses et d'élaborer une action proportionnée, distanciée. » (p. 95). Assurément, dit l'auteur, les catégories sont des synthèses réductrices, mais le problème se manifeste quand elles dirigent trop facilement vers « des actions réflexes là où, au contraire, il faudrait de la réflexion» (p. 90). Et quand, non seulement les mises en scène médiatiques et politiques font un usage immodéré de ces simplifications, mais aussi quand les constructions intellectuelles n'arment pas pour les dépasser.

Pour lui, la thématique de la souffrance au travail, qui «nous fait entrer dans un monde de souffrance peuplé de deux catégories, les victimes qui souffrent et les bourreaux qui font souffrir » (p. 97), l'illustre à l'extrême. Les catégories utilisées (violence au travail, harcèlement moral, souffrance...) ne nous sortent pas des bipartitions entre le bien et le mal, n'équipent pas une controverse où, sur le terrain de la gestion, on pourrait ouvrir le débat sur l'existence d'une pluralité de catégorisations possibles. De telles catégories servent les experts en « risques psychosociaux », qui savent ainsi se rendre indispensables, plutôt qu'elles n'aident les salariés et leurs représentants à construire par euxmêmes des controverses sur l'organisation et la gestion. Non sans devoir faire évoluer le sens de ce qu'on entend par organisation du travail. Dans les services, avec la relation de service et les « tensions servicielles », ce sens se renouvelle: c'est être équipé pour réguler ces tensions.

Les violences vécues dans les services publics ou, parfois, « simplement», les incivilités ou les agressions verbales constituent justement l'objet du chapitre 5 . Le pilotage à distance et les outils normalisateurs y font des dégâts, mis en discussion par l'auteur autrement qu'en termes de souffrance. Les cas où l'on contient la violence sont ceux où, dans des antennes de proximité, la productivité est moins comptabilisée, où l'on accepte le coût budgétaire de cet ajustement sur place. «Contenait », faudrait-il dire, car l'auteur a vu ces formes organisationnelles avoir tendance à être remplacées par solutions centralisées et standard, par exemple une plate-forme d'appel. La violence naît d'autant plus volontiers dans les services que l'on applique dans ces activités les séparations qui valaient dans l'industrie entre conception, distribution et production. Un centre concepteur de l'offre de service, où l'on édicte ce qui est bon pour la relation de service, à distance, et, de l'autre, « le face-à-face réel » des professionnels de première ligne avec les usagers en chair et en os (p. 117), recevant les prescriptions multiples et non accordées de toute une diversité de parties prenantes. Quand le cadre 
est défini une fois pour toutes et à distance de ces contextes, les professionnels n'ont plus de marges pour réguler les tentatives diverses de prescrire: pour s'accorder, professionnels et usagers manquent de temps, de ressources (par exemple culturelles, pour certains usagers), de soutien, notamment du collectif, pour certains professionnels. "Trop de cadrage standard, pas assez de ressources pour s'ajuster, et le sentiment réciproque de frustration » (p. 119).

Ainsi des catégories de gestion exposent les salariés à des risques quand elles ne font pas concevoir que réguler les tensions se passe d'autant mieux que le collectif de travail bénéficie d'une certaine stabilité, de conditions pour constituer, accumuler, transmettre, faire évoluer de l'expérience, individuelle et collective.

Or, défend le chapitre 6 , il y a, aujourd'hui, « frénésie gestionnaire », résultat d'une prolifération des enjeux de gestion, d'une accentuation de la pression du résultat et d'une accélération du rythme des changements et réformes. Les suicides professionnels illustrent, aux yeux de F. Ginsbourger, la tendance de la gestion, dans ce cadre, à devenir perverse, au sens où elle place les personnes « dans une situation à proprement parler ingérable » (p. 137). Dans les organisations contemporaines, les instruments de gestion se répandent, toujours plus nombreux, mais souvent «à côté de la plaque », nous dit l'auteur. Ils enferment les individus dans des actions hors de propos par rapport à ce qu'ils estiment nécessaire. Les organisations sont devenues, ces dernières années, des lieux où, quand on est en désaccord avec ce qu'on nous demande de faire, sans entrevoir pour autant les possibilités de l'exit, de la voice ou de la loyauté, il n'y a plus d'autre solution que de tricher avec le système, dire noir quand on pense blanc, etc. Les « risques psychosociaux », que beaucoup interprètent comme une souffrance résultant de la dictature de la finance, du profit et de la gestion, ont, pour notre auteur, directement à voir, en fait, avec des méthodes comme les mobilités forcées et la « mise en inconfort » (i.e. la tentative pour que les individus partent d'eux-mêmes, écœurés), bref toute une organisation de l'obligation faite aux salariés de « se contorsionner dans tous les sens », de « tricher avec le système »: « Ne renoncer ni à son métier, ni à son statut, ni à l'entreprise, ni à une activité, se maintenir contre vents et marées, essayer de tenir malgré tout, de passer entre les mailles des filets. » (p. 147). «France Télécom est emblématique de la perversité qui se niche dans le clivage, spécifiquement français, entre un haut niveau de protection de l'emploi et un faible niveau de régulation de l'organisation du travail » (ibid.).

Le chapitre 7 clôt l'ouvrage en invitant les acteurs sociaux et politiques à « apprendre la critique de la gestion », en distinguant cela de la dénonciation courante de la tyrannie du management et en ancrant la réflexion dans l'économie de services contemporaine.

Il faut lire l'ouvrage de Francis Ginsbourger et prendre le temps de saisir toute l'importance et la réelle signification de son entrée par les « catégories ».

D'abord, l'alternative ainsi échafaudée conteste et se substitue à celle de la « financiarisation ». En cela, l'ouvrage est à rebours de la plus grande part des analyses à disposition, qui ressortent de l'ouvrage comme procédant d'une volonté d'interprétation trop pressée.

Ensuite, les catégories sont produites... non dans la seule sphère de l'entreprise mais dans des vaet-vient avec les divers univers de ce que l'auteur nomme les «professionnels de la connaissance». C'est au fond un postulat très latourien... et très sain : alors que, chacun à sa manière, les chercheurs, les journalistes, les consultants, les experts de toutes sortes ont tendance à se concevoir en extériorité et en surplomb, l'auteur fait comprendre qu'ils font bien partie du même univers socio-technique que les acteurs sociaux et donc de l'histoire en train de se jouer. Ils contribuent, et comment, à l'élaboration des catégories qui viennent, ensuite, influencer le réel. Ils ont donc une responsabilité. Celle d'ouvrir ou de fermer des voies d'action, de mettre en lumière ou dans l'obscurité des leviers pour agir.

Il serait faux, pour le chercheur, de lire l'ouvrage comme étant celui « d'un consultant ». C'est dans le petit monde des universitaires que l'on se voit, chercheur, radicalement différent des consultants. Quand on adopte comme échelle d'observation celle des catégorisations et des professionnels de la 
production et diffusion de celles-ci, il y a beaucoup plus de points de communication entre ces sousunivers professionnels. Tous les professionnels de la connaissance ont une responsabilité.

C'est cette échelle qui permettra de comprendre ce qui pourrait, rapidement, être évacué d'un revers de main comme des amalgames. En particulier, les tenants de la psychodynamique du travail n'apprécieront certainement pas de voir confondus la thèse de la souffrance de Christophe Dejours, le rapport Nasse-Légeron et les diagnostics du cabinet Technologia. Effectivement, si on est un lecteur pressé, il y a là, de la part de l'auteur, une confusion inacceptable. Avec un centrage de la focale plus serré, on devrait, évidemment, introduire plus de nuance ou de distinctions que ne le fait l'auteur. Le rapport Nasse-Légeron relève d'une épistémologie complètement opposée à celle de C. Dejours, et F. Ginsbourger ne l'ignore assurément pas. Aussi son propos n'est-il pas là. C'est d'appeler chacun à estimer qu'il y a une responsabilité partagée à susciter le développement de catégories qui, par la dramatisation, par le vocabulaire du risque et de la victime, dénoncent la gestion au lieu de porter les acteurs gestionnaires et les représentants de salariés à reconstruire une capacité à interroger l'organisation dans ce qu'elle fait au travail: enfermer les salariés dans des situations intenables ou les équiper dans la consolidation d'apprentissages où, à la fois, ils se rendent capables d'affronter les situations difficiles et créent de la valeur. Dans un même univers sociotechnique, des catégories de chercheurs qui n'ouvrent pas de controverses sur les catégories de gestion mais sur une dénonciation façonnent le terrain sans, au minimum, contrarier l'avancée des expertises techniques telles que les diagnostics de risques psychosociaux.

Combatif, l'ouvrage (qui en agacera certains, cela est sûr) mérite à tout prix le détour. Limpide, d'une écriture à l'accessibilité louable, il n'en doit pas moins faire l'objet d'une lecture patiente, attentive à ne pas lire autre chose que ce qui est écrit. Car l'auteur n'est peut-être pas à l'abri du risque que des lecteurs l'abordent en conservant à l'esprit les catégories les plus courantes pour le comprendre. Ce serait une mésaventure bien malencontreuse. 